\title{
TEM Observation of Dislocation Emission from a Crack at DBTT in Si
}

\author{
Suprijadi* and Hiroyasu Saka \\ Department of Quantum Engineering, Nagoya University, Nagoya 464-8603, Japan
}

Emission of dislocations from a crack which propagated at the ductile-brittle transition temperature (DBTT) in Si was observed by combined use of focused-ion beam (FIB) technique and transmission electron microscopy (TEM). At the wake of a DBTT crack many dislocation lines and dislocation loops were observed, while a wake of a precrack introduced at room temperature, no dislocations were observed. In addition, those glide dislocation lines which are emitted at the DBTT crack are smoothly curved, indicating that they can overcome easily the Peierls stress.

(Received August 4, 2000; Accepted September 25, 2000)

Keywords: ductile-brittle transition, transmission electron microscopy, focused ion beam, dislocation, crack tip, silicon

\section{Introduction}

Ductile-brittle transition of materials is of particular interest in materials science. Rice and Thomson ${ }^{1)}$ proposed an idea of competition between shear and cleavage failure. They considered that the necessary condition for ductile fracture is the spontaneous emission of a dislocation loop from a cleavage crack. Following this idea, a number of investigations attempt to analyze the stress and displacement fields of a dislocation near a crack tip. ${ }^{2,3)}$ Simulation of crack propagation on atomic scale ${ }^{4)}$ shows the emission of dislocations at a mode I crack; the dynamic process of dislocation emission, including the nucleation from the crack tip and the motion away from the crack was demonstrated.

Evidence for the emission of dislocations has been collected using etch-pitting technique, ${ }^{5)} \mathrm{X}$-ray topography ${ }^{6)}$ and, to less extent, transmission electron microscopy (TEM). ${ }^{3,7-9)}$ The bottleneck for TEM observation of dislocation emission at a crack tip is related with the difficulty in preparing a thin foil specimen from a pre-selected area. However, this difficulty was overcome by applying a focused-ion beam (FIB) technique to preparation of thin foil specimens. ${ }^{10-12)}$

In the present study, emission of dislocations at a crack tip

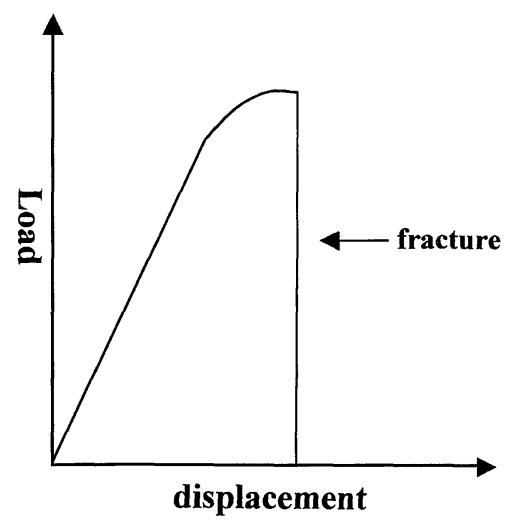

Fig. 1 A schematic illustration of a load-displacement curve obtained on $\mathrm{Si}$ in a three-point bending test at DBTT.

${ }^{*}$ Permanent address: Department of Physics, Institute of Technology Bandung, Jl. Ganesha no: 10, Bandung 40132, INDONESIA. at DBTT in Si was studied by combined use of FIB and TEM and possible mechanisms for dislocation emission were discussed.

\section{Experimental Procedures}

Plate shaped dislocation free Si single crystals were used. Vickers indentations were performed on a mirror plane of
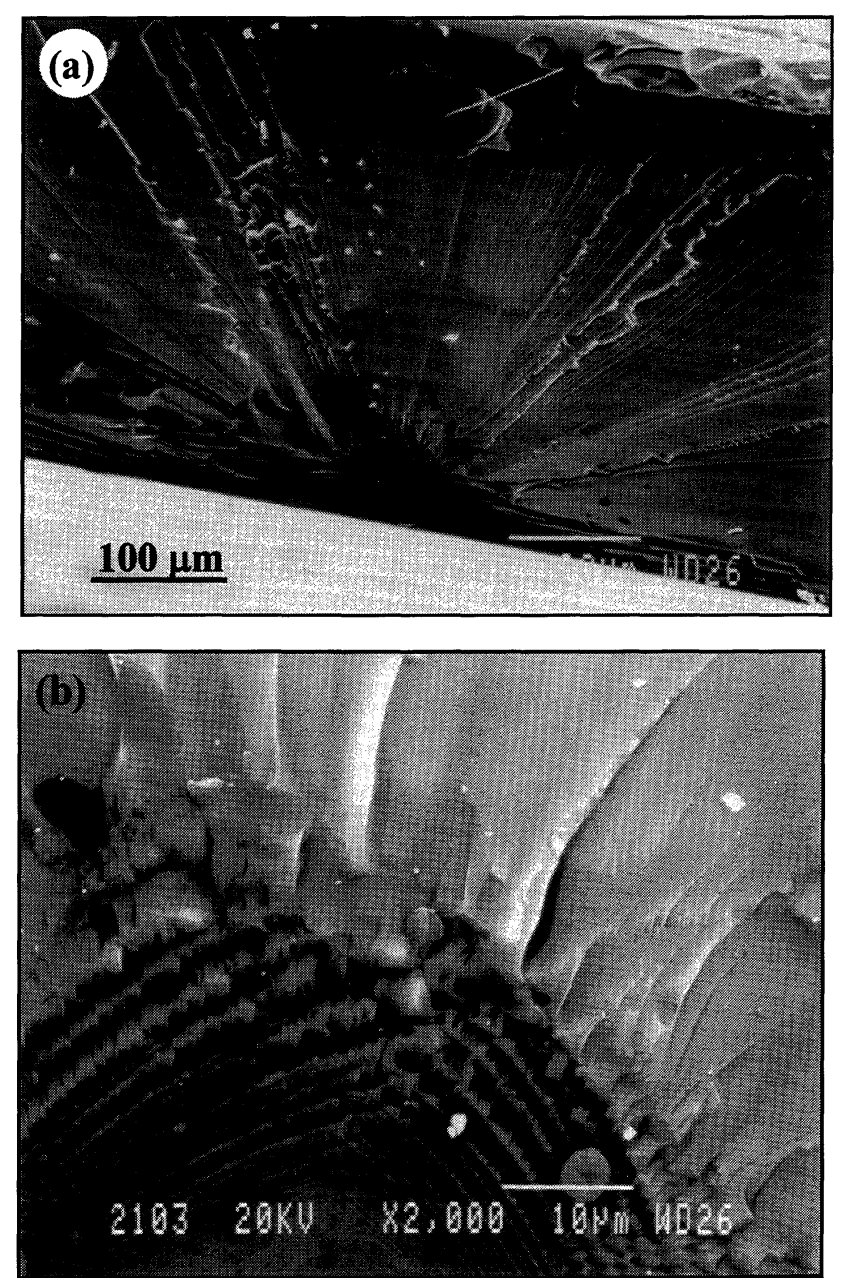

Fig. 2 (a) Low magnification SEM micrograph of the fracture face of $\mathrm{Si}$ deformed to fracture at DBTT (b) Details of the fracture face near the precrack front. 
(001) with a load of $0.49 \mathrm{~N}$ and a dwell time of $5 \mathrm{~s}$ at room temperature. Cracks lying on $\{110\}$ planes were introduced. Then, the pre-cracked samples were bent in three-point bending $^{12)}$ at $873 \mathrm{~K}$, which is close to DBTT in such a way that the one of the two pre-cracks serves as a precursor of failure. Some samples were deformed into fracture, while some were deformed to a stress of about $80 \%$ of the fracture stress. Fracture faces were examined in a JEOL scanning electron microscope. Two types of foil specimens for TEM observation were prepared using a focused ion beam technique. In one type, the crack which propagated under the action of an external stress at DBTT was almost perpendicular to the foil surface, while, in the other, the fracture surface coincides with one of the surfaces of the foil specimen. Hereinafter the former type of the specimens will be called cross-sectional specimens and the latter plan-view ones. These specimens were

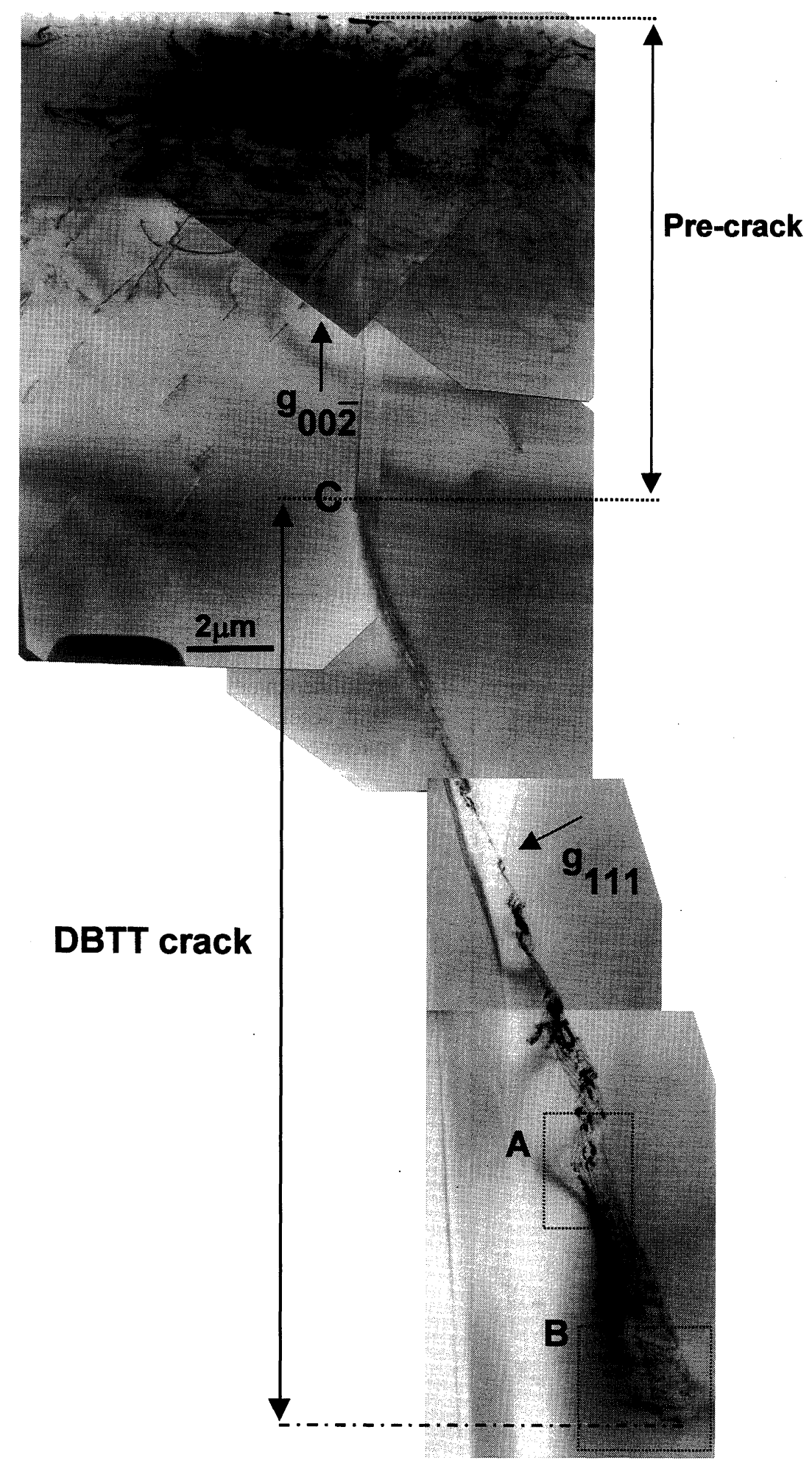

Fig. 3 An example of propagation of a crack at DBTT. Precrack, introduced at room temperature, is very straight, while DBTT crack, which propagated at DBTT, is zigzagged. 


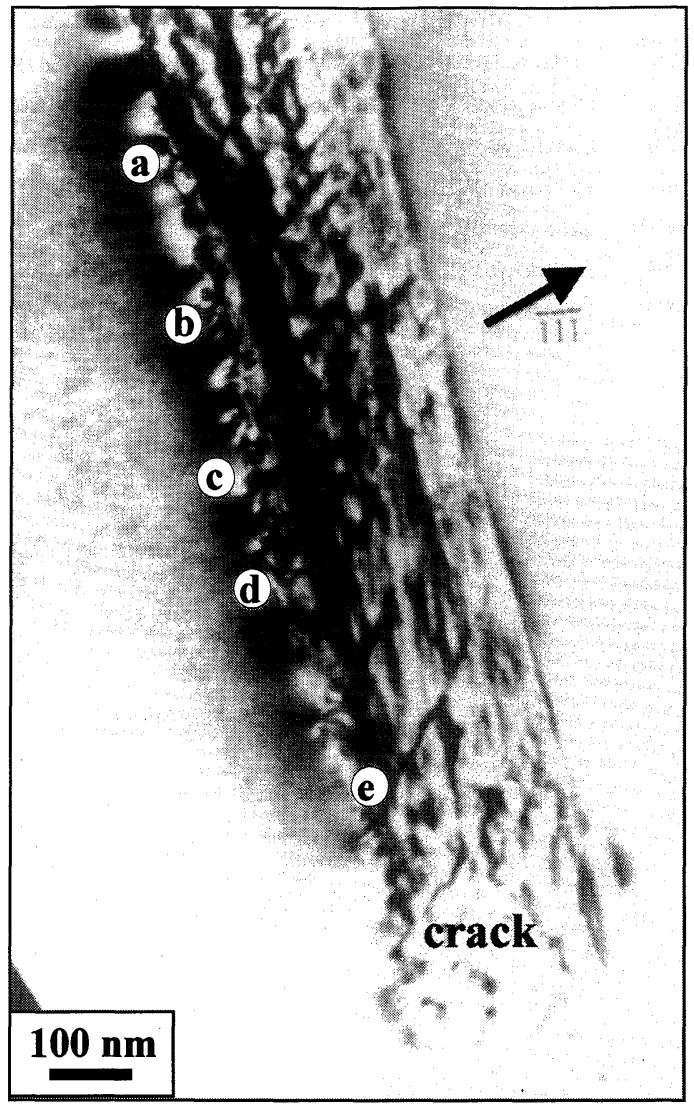

(a)

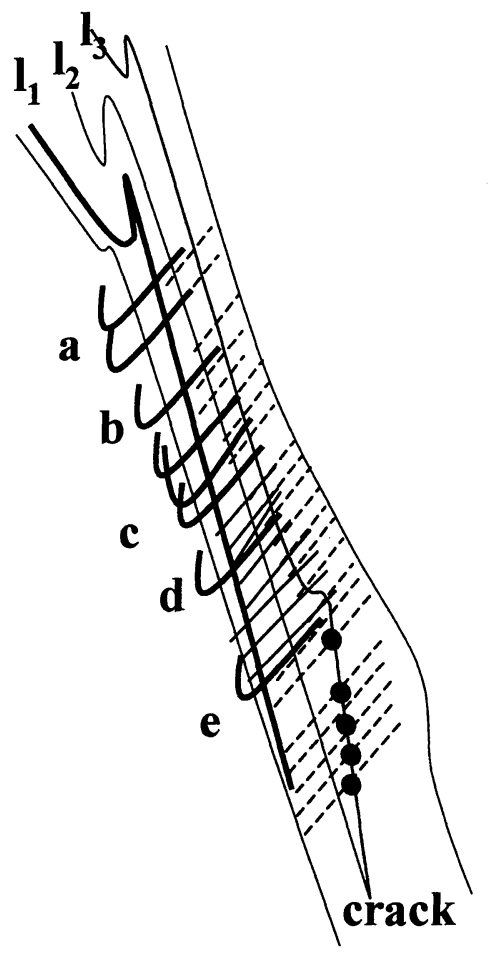

(b)

Fig. 4 (a) Details of area denoted by A in Fig. 3. (b) Schematic illustration of (a).

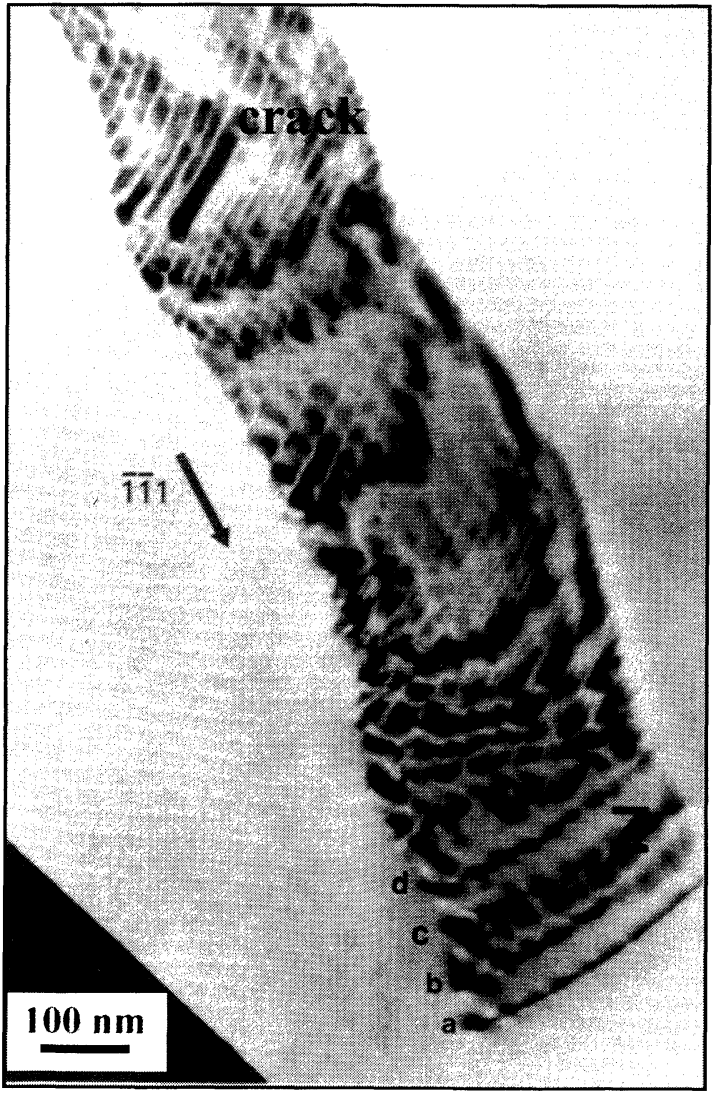

Fig. 5 Details of the crack tip, area denoted by B in Fig. 3. examined in either a Hitachi HU-1000D microscope operated at an accelerating voltage of $1000 \mathrm{kV}$ or a Hitachi NAR-9000 microscope operated at $300 \mathrm{kV}$.

\section{Results}

\subsection{Load-displacement curves at DBTT and SEM obser- vation}

Figure 1 shows a typical example of the load-displacement curve obtained in a three-point bending test at DBTT. After the linear elastic deformation, the specimen deformed plastically to some extent, and then catastrophically fractured.

Figure 2(a) shows a low-magnification SEM micrograph of a fracture face after the pre-cracked specimen was deformed to fracture at DBTT. The origin of the failure was the precrack introduced at room temperature. The face of the precrack is very flat. A series of radial fan-like markings that begin at the precrack front extend outwards to the specimen boundaries. Figure 2(b) shows the area near the precrack front at a higher magnification. In the area between the precrack front and the radial fan-like markings, fine linear dome-like markings run perpendicularly to the propagation direction of the crack front. The spacing and the amplitude of the linear markings increases systematically from a few $\mathrm{nm}$ to a few microns with increasing the distance from the precrack front. It is suggested that propagation of the crack over the domelike and fan-like areas corresponds to the plastic deformation and fracture, respectively, in the load-displacement curve in 


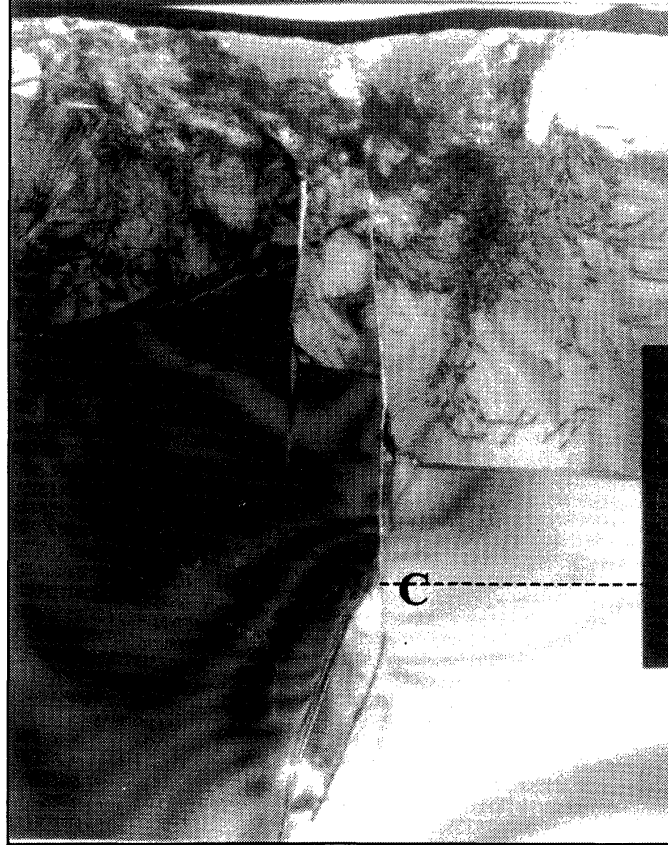

(a)

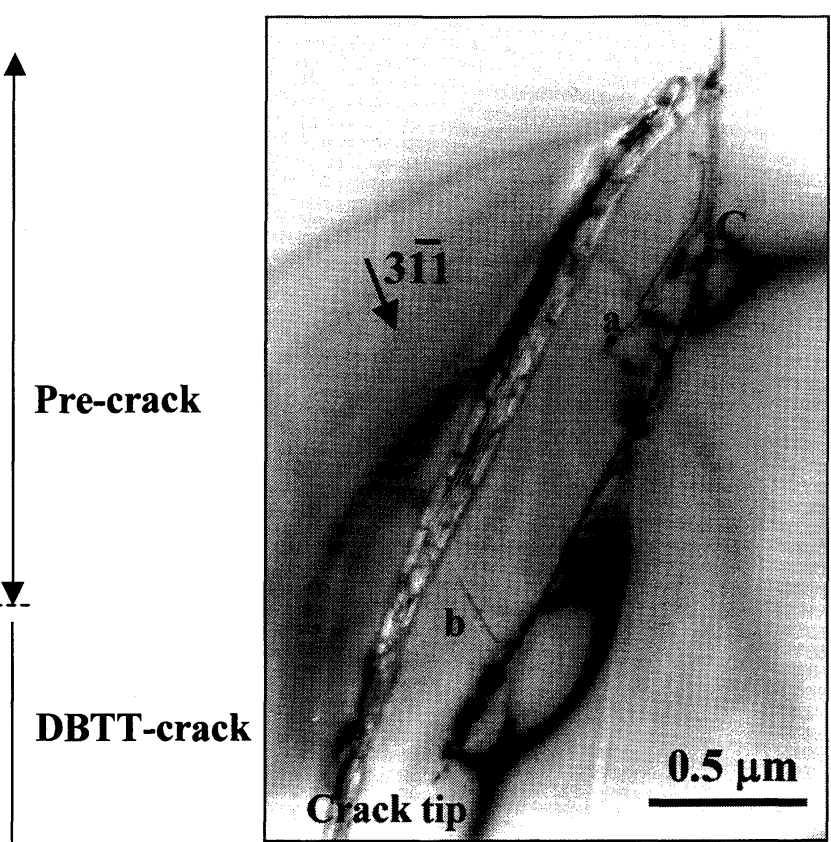

(b)

Fig. 6 (a) Low-magnification micrograph showing another example of propagation of cracks at DBTT. (b) Detail of area denoted by C in (a). A precrack is bifurcated into two DBTT cracks. Dislocations a and b are perfect dislocations which must have been emitted during propagation of the DBTT cracks.

Fig. 1.

\subsection{Cross-sectional TEM observation}

Figure 3 shows an example of propagation of a crack at DBTT. The precrack, introduced at room temperature, was very straight (from the free surface to $C$ ) but the crack which propagated at DBTT (hereinafter called DBTT crack) started deviating from the $\langle 110\rangle$ and became zigzagged. ${ }^{12)}$ The spacing and the amplitude of zigzagging increases systematically from a few nm near the precrack front with increasing the distance from the precrack front. Thus, this zigzagging corresponds exactly to a cross-section of the dome shown in Fig. 2(b). Furthermore, no dislocations were observed at the wake of the precrack but many observed at the wake of the DBTT crack.

Figure 4(a) shows a part of the DBTT crack (denoted A in Fig. 3) at high magnification. It is evident that many dislocations are observed near the wake of the DBTT crack, but it is not clear whether they are perfect dislocations or the stacking fault. At least some dislocations were identified to have been emitted from the fracture face, as shown schematically in Fig. 4(b).

Figure 5 shows detailed structure near the tip of the DBTT crack (denoted B in Fig. 3). Many linear markings, most probably dislocations, are observed at the fracture faces and at least 4 dislocations a, b, c and d are observed to have been emitted at the crack tip.

Figures 6(a) and 6(b) show another example of propagation of a crack at DBTT. In this case, the precrack is decomposed into two DBTT cracks. Between the two DBTT cracks perfect dislocations $a$ and $b$ are observed; they must have been emitted during the propagation of the DBTT cracks.

\subsection{Plan-view TEM observation}

Figure 7(a) shows defect structure in the sub-surface area just underneath the DBTT crack, taken in $\boldsymbol{g}=[220]$ reflection vector. Two cracks $A$ and $B$ have been bifurcated from a main crack, as schematically shown in Fig. 7(b). Crack A shows Moire fringes, suggesting that it remains a crack, while crack B contains dislocation networks, suggesting that it has been healed. Many dislocations are emitted from the dislocation network on crack B. All of them are smoothly curved.

\section{Discussion}

Many dislocations are observed at the wake of a DBTT crack. Possible mechanism for the formation of such dislocations has been proposed. ${ }^{12)}$ They assumed that the propagation of a mode I crack gives rise to supersaturation of point defects, i.e., vacancies and/or interstitials. At DBTT supersaturated point defects agglomerate to form dislocation loops, which act as dislocation sources. On the other hand, below DBTT, mobility of point defects is too small for the dislocation loops to be formed as a result of the agglomeration of the point defects.

However, when a crack is introduced by indentation, the crack eventually is halted at some place where the stress due to indentation is no longer strong enough to drive the crack to propagate. In other words, the velocity of the crack must have been very small just behind the crack front. Under such circumstances, some of the supersaturated point defects may agglomerate to form clusters even at room temperature. Indeed, evidence for such a defect cluster has been observed by Saka and Nagaya. ${ }^{10)}$

Another finding of the present study is that dislocations 

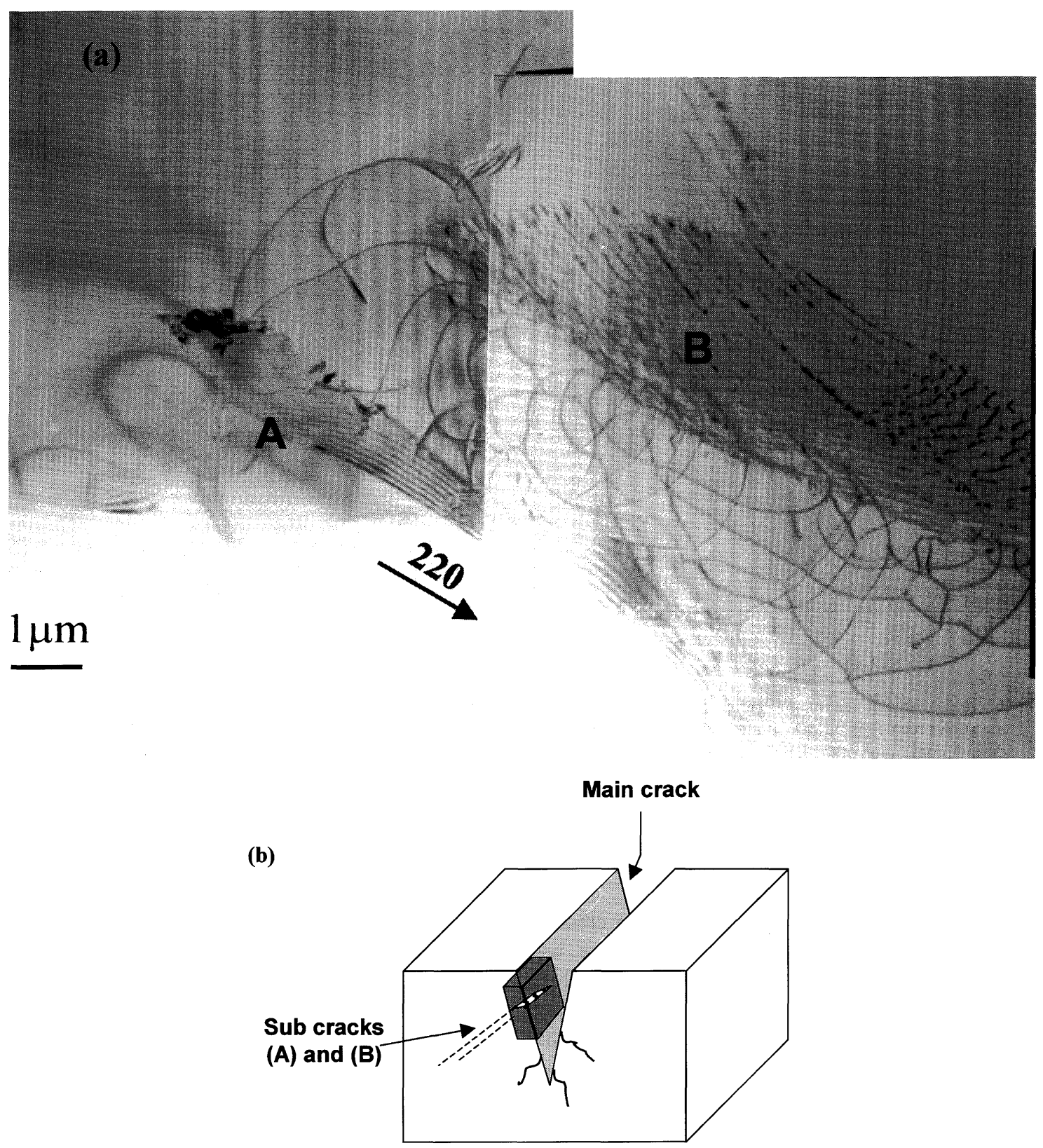

Fig. 7 (a) Plan-view observation of the defect structure in the sub-surface area just underneath the DBTT crack. Many dislocations have been emitted during propagation of a DBTT crack. All of them are smoothly curved. (b) Sub-crack schematic diagram.

emitted at DBTT in Si are smoothly curved (Fig. 7). It is well known that dislocations in Si experience a very high Peierls stress. Thus, dislocations in Si tend to lie along $\langle 110\rangle$ directions, taking a shape of a hexagon. ${ }^{13)}$ This can be explained by taking into account that the stress at which dislocations start moving at DBTT in Si is very high (see Fig. 3). Once dislocations start moving, they can easily overcome the Peierls stress.

\section{Conclusions}

Combination of FIB and TEM allows one to observe preselected areas around a crack. In the present study, relationship between morphology of a fracture face and the dislocation structure underneath was established by this new method.

\section{REFERENCES}

1) J. R. Rice and R. Thomson: Philos. Mag. 29 (1974) 73-97.

2) R. Thomson. and A. E. Carlsson: Philos. Mag. A70 (1994) 893-903.

3) B. R. Majumdar and S. J. Burns: Scr. Metall. 14 (1980) 653-565.

4) F. Cleri, S. Yip, D. Wolf and S. R. Phillpot: Phys. Rev. Lett. 79 (1997) 1309-1312.

5) J. Samuels and S. G. Roberts: Proc. Roy. Soc. London A421 (1989) $1-23$.

6) A. George and G. Michot: Materi. Sci. \& Engg. A164 (1993) 118-134.

7) B. R. Lawn, B. J. Hockley and S. M. Wiederhorn: J. Mater. Sci. 15 (1980) 1207-1223.

8) Y. H. Chiao and D. R. Clarke: Acta Metall. 37 (1989) 203-219.

9) K. Higashida, N. Narita, R. Onodera, S. Minato and S. Okazaki: Mater. Sci. Eng. A273 (1997) 72-78.

10) H. Saka and G. Nagaya: Philos. Mag. Lett. 72 (1995) 251-255.

11) H. Saka and S. Abe: J. Electron Microsc. 1 (1997) 45-57.

12) Suprijadi and H. Saka: Philos. Mag. Lett. 78 (1998) 435-443.

13) K. Wessel and H. Alexander: Philos. Mag. 35 (1977) 1523-1536. 